\section{INTERCHANGEABILITY OF PD-L1 LABORATORY- DEVELOPED TEST BY 22C3 ANTIBODY CONCENTRATE AMONG IHC PLATFORMS IN GASTRIC CANCER}

Jimin Kim ${ }^{1,2}$, Binnari Kim ${ }^{1,2}$, Eunji Kim², Minsun Jang ${ }^{2}$, Jun Hun Cho ${ }^{1}$, Hye Seung Lee ${ }^{3}$, Yoonjin Kwak ${ }^{4}$, Lingkang Huang ${ }^{5}$, Jonathan Juco ${ }^{5}$, Sally Bai ${ }^{5}$, Kyoung-Mee Kim ${ }^{1,2}$

${ }^{1}$ Department of Pathology and Translational Genomics, Samsung Medical Center, Sungkyunkwan University School of Medicine, Seoul, Republic of Korea; ${ }^{2}$ Center of Companion Diagnostics, Samsung Medical Center, Seoul, Republic of Korea; ${ }^{3}$ Department of Pathology, Seoul National University Bundang Hospital, Bundang, Republic of Korea; ${ }^{4}$ Department of Pathology, Seoul National University College of Medicine, Seoul, Republic of Korea; and ${ }^{5}$ Merck \& Co., Inc., Kenilworth, $N J, U S A$

Background: The PD-L1 IHC $22 \mathrm{C} 3$ pharmDx assay on the Dako Autostainer Link 48 (ASL48) platform has been established to measure PD-L1 expression in gastric cancer (GC); however, availability of this platform is limited. We developed an IHC laboratory-developed test (LDT) using the $22 \mathrm{C} 3$ antibody concentrate to determine PD-L1 expression in patients with GC.

Methods: PD-L1-stained tumour specimens $(N=120)$ from patients with GC or GEJ adenocarcinoma were scored for combined positive score (CPS) $\geq 1$ by 3 pathologists at 3 sites. The PD-L1 IHC 22C 3 pharmDx assay on the Dako ASL48 served as the gold standard; the $22 \mathrm{C} 3$ antibody concentrate was tested on 3 platforms: Dako ASL48, Ventana BenchMark Ultra, and Leica Bond-Max.

Results: Intraclass correlation coefficient of CPS as a continuous variable between the gold standard and each platform ranged from 0.910-0.989. When CPS was dichotomised based on a score $\geq 1$, the total agreement ranged from $87-98 \%$, positive percentage agreement ranged from $81-99 \%$, and negative percentage agreement ranged from 90-100\%. Intra-pathologist concordances using the $22 \mathrm{C} 3$ pharmDx assay also showed substantial agreement (kappa value 0.779).

Conclusions: The $22 \mathrm{C} 3$ antibody concentrate can be successfully used on 3 IHC platforms to determine PD-L1 expression in tumour samples from patients with GC.

\section{PTEN PROTEIN LOSSES AND LOSS-OF-FUNCTION GENETIC VARIANTS IN GASTRIC CANCERS: THE RELATIONSHIP WITH MICROSATELLITE INSTABILITY, EBV, AND PD-L1 EXPRESSION}

Binnari Kim ${ }^{1,2}$, So Young Kang ${ }^{1}$, Deokgeun $\mathrm{Kim}^{3}$, Eun Ji Kim ${ }^{2}$, You Jeong $\mathrm{Heo}^{4}$, Kyoung-Mee Kim ${ }^{1,2}$ ${ }^{1}$ Department of Pathology and Translational Genomics, Samsung Medical Center, Sungkyunkwan University School of Medicine, Seoul, Republic of Korea; ${ }^{2}$ Center of Companion Diagnostics, Samsung Medical Center, Seoul, Republic of Korea; ${ }^{3}$ Center of Clinical Genomics, Samsung Medical Center, Seoul, Republic of Korea; and ${ }^{4}$ The Samsung Advanced Institute for Health Sciences \& Technology (SAIHST), Samsung
Medical Center, Sungkyunkwan University School of Medicine, Seoul, Korea

Background: Inactivation of PTEN is caused by various mechanisms and is related to progression of cancer. In gastric carcinoma (GC), the relationship between PTEN protein losses and genetic variants is unclear and their effects on microsatellite instability (MSI), EBV, and PD-L1 expression are not studied. Methods: We performed comprehensive cancer panel tests with 322 tumour tissues from advanced GC patients. In all cases, immunohistochemistry for PTEN protein was performed, and loss of protein expression is defined as a complete absence of nuclear staining.

Results: In total, 34 cases (10.6\%) had PTEN mutations and 13 of them (55.9\%) showed PTEN protein losses. In 288 wild-type GC for PTEN, protein losses were found in 35 cases $(12.2 \%)$. PTEN protein losses were significantly associated with PTEN mutations $(p=5.232 \mathrm{e}-10)$, MSI-high $(p=3.936 \mathrm{e}-08)$, and EBVnegativity $(p=0.0071)$. The most common PTEN variants with protein losses were p.R130 found in 4 cases, p.R335 in 2 cases, and all cases with nonsense mutation showed PTEN inactivation. Conclusions: PTEN loss-of-function mutation is an important genetic mechanism of PTEN inactivation.

\section{NON-STANDARDISED REPORTING OF AUTOANTIBODY ASSAYS AMONGST AUSTRALIAN AND NEW ZEALAND LABORATORIES: RESULTS OF A SURVEY}

Matthew Krummenacher, Pravin Hissaria

SA Pathology and Royal Adelaide Hospital, Adelaide, Australia

Background: Autoantibody results only slightly above established cut-offs are considered to have lower specificity and provide lower likelihood ratios; their clinical significance may therefore be uncertain, which is not readily communicated with dichotomous qualitative reporting.

Line immunoblots have become a common method for detecting autoantibodies due to a streamlined methodology. However, the manufacturer's recommended positive cut-off, which is uniform for all antibodies, is contentious.

Methods: We distributed a survey via e-mail to Australasian immunopathology laboratories to investigate how autoantibody assays are currently being interpreted and reported, with a focus on line immunoblots. The survey was developed using www. surveymonkey.com.

Results: There were 31 responders, comprising immunopathologists and scientists from at least 17 unique laboratories across Australia (8 public, 5 private) and New Zealand (4 laboratories). Autoantibody reporting was not standardised; there were significant differences in reporting practices, particularly regarding the interpretation of and positive cut-offs used for line immunoblots, which were often contrary to manufacturer's guidelines. Interpretative qualitative reporting based on results from other investigations and clinical history was a common theme.

Conclusions: There is a need for standardised guidelines regarding reporting of autoantibody assays. A collaborative effort from immunopathologists, clinicians and industry is required. Assay manufacturers must justify recommended cutoffs. 